\title{
COMPRENSIÓN, DISTANCIAMIENTO E INTERVENCIÓN: DESAFÍOS METODOLÓGICOS EN UNA INVESTIGACIÓN SOBRE MIGRACIÓN Y EDUCACIÓN
}

\author{
Gabriela Novaro ${ }^{1}$
}

\begin{abstract}
Resumen
En este artículo sistematizo algunas reflexiones metodológicas a partir de una investigación de corte cualitativo etnográfico en el campo de la migración y la educación. Advierto la importancia de la continuidad del trabajo y la permanencia en el territorio para acceder a las experiencias de los distintos actores y a los sentidos locales de categorías que en mi caso resultan nodales (descendientes, segunda generación, seguir siendo bolivianos, ser alguien). Me detengo a continuación en las potencialidades de la etnografía para comprender y a la vez tomar distancia de esos significados. Para finalizar reflexiono sobre las potencialidades y riesgos de la complementación de actividades más tradicionales asociadas a la investigación cualitativa (observación, entrevistas, reconstrucción de trayectorias biográficas) con experiencias de intervención que he venido sosteniendo en la localidad: talleres en escuelas, acompañamiento de proyectos de alfabetización, participación en radios comunitarias.
\end{abstract}

Palabras clave: Metodología. Enfoque etnográfico. Reflexividad. Distanciamiento. Intervención.

\section{COMPREHENSION, DISTANCING AND INTERVENTION: METHODOLOGICAL CHALLENGES IN AN INVESTIGATION ABOUT MIGRATION AND EDUCATION}

\section{Summary}

In this article there are systematize some methodological reflections starting from an investigation of qualitative ethnographic type in the field of migration and education. It is warned about the relevance of the continuity of the work and the permanency in the territory to acquire the experiences of the different subjects and the local senses that in this case result nodal (descendants, second generation, continue being Bolivians, to become someone). Then it is stopped in the potentialities of the ethnography to comprehend and at the same time to establish a distance to those meanings. To finalize it is pondered upon the potentialities and the risks of the complementation of more traditional activities related to the qualitative investigation (observation, interviews, reconstruction of biographical trajectories) with experiences of intervention that it was sustained in the locality: workshops in schools, accompaniment in projects of alphabetization and participation in community radio programs.

Keywords: Methodology. Ethnographic approach. Reflexibility. Distancing. Intervention.

\footnotetext{
${ }^{1}$ Investigadora del Consejo Nacional de Investigaciones Científicas y Técnicas - Profesora de Antropología de la Universidad de Buenos Aires - Doctora em Antropología - gabriela.novaro@gmail.com
} 


\section{COMPREENSÃO, DISTANCIAMENTO E INTERVENÇÃO: DESAFIOS METODOLÓGICOS EM UMA INVESTIGAÇÃO SOBRE MIGRAÇÃO E EDUCAÇÃO}

Neste artigo são sistematizadas algumas reflexões metodológicas baseadas na pesquisa etnográfica qualitativa no campo da migração e da educação. Percebe-se a importância de continuar trabalhando e permanecendo no território para acessar as experiências de diferentes atores e os significados locais de categorias que no meu caso resultam em nodais (descendentes, segunda geração, vá em frente, seja alguém). Para-se na continuação das potencialidades da etnografia para compreender e ao mesmo tempo me distanciar desses significados. Para finalizar, reflito sobre as potencialidades e riscos de complementar atividades mais tradicionais associadas à pesquisa qualitativa (observação, entrevistas, reconstrução de trajetórias biográficas) com experiências de intervenção sustentadas na localidade: oficinas em escolas, acompanhamento de projetos de alfabetização, participação nas rádios comunitárias.

Palavras-chave: Metodologia. Abordagem etnográfica. Reflexividade. Distanciamento. Intervenção.

\section{Introducción}

En este artículo me propongo reflexionar sobre los desafíos y potencialidades metodológicas de un trabajo de investigación etnográfica sobre migración y educación.

Comienzo presentando la localidad donde trabajo y las características generales de una investigación de muchos años que ha implicado la complementación de herramientas metodológicas y el trabajo colaborativo con otros colegas. Me detengo luego en tres cuestiones que el enfoque etnográfico posibilita: la atención a los sentidos locales de las categorías, la diferenciación entre la comprensión de las situaciones que estudiamos y la adhesión a las posiciones de los distintos actores, la inclusión de actividades de intervención y la reflexividad sobre las mismas como parte del diseño de investigación.

Recupero en primer lugar la tradición de la antropología para considerar los sentidos sociales que se presentan en el trabajo de campo. Mi interés por la formación e identificación de las jóvenes generaciones me ha llevado a atender en particular a nociones como descendientes. seguir siendo, ser alguien, bolivianos de segunda generación, sangre boliviana. Desde esta recuperación planteo la necesidad de enriquecer lo que entendemos por educación, formación, relaciones generacionales, identificaciones nacionales.

A continuación reflexiono sobre la necesidad de diferenciar la comprensión de los procesos de la adhesión a sus sentidos, sobre todo en situaciones donde no coincidimos con las posiciones de aquellos con quienes trabajamos e incluso estas posiciones incomodan nuestros presupuestos. En mi investigación advierto esto en cuestiones como la expectativa de mayor disciplinamiento que las familias realizan a las escuelas y las representaciones de los actores escolares sobre las familias bolivianas.

Para finalizar me detengo en la potencialidad del desarrollo de actividades no previstas pero que fueron surgiendo como respuesta a las demandas de distintos actores de la localidad: la colaboración en clases escolares, en un programa de alfabetización de un centro de mujeres y en una radio comunitaria. Estas actividades, además de dar respuesta a pedidos concretos y 
afianzar las relaciones con distintos sujetos, fueron fundamentales para precisar las categorías en uso en la localidad y profundizar la comprensión de las prácticas y relaciones.

Las temáticas señaladas de ninguna manera agotan los innumerables desafíos metodológicos que encuentran nuestros trabajos; representan sin embargo tres cuestiones claves para considerar tanto los condicionantes de la labor etnográfica, como sus potencialidades.

\section{La localidad y el trabajo de campo: permanencia, complementación de herramientas y trabajo colectivo}

Desde el 2010 desarrollo mi investigación en un barrio de la localidad de Escobar ubicado $50 \mathrm{~km}$ al norte de la Ciudad de Buenos Aires-Argentina. El barrio está habitado en una proporción significativa por migrantes procedentes de Potosí- Bolivia y por sus descendientes; se caracteriza por el fuerte peso de las organizaciones, en particular la Colectividad Boliviana de Escobar (en adelante la CBE). Esta entidad mantiene fuertes asociaciones con el Estado boliviano y con el gobierno local, sostiene actividades de acopio y comercialización de productos hortícolas, organiza eventos festivos y recreativos y tiene además una radio comunitaria. Los jóvenes de familias migrantes se incluyen en la CBE sobre todo en el trabajo en puestos familiares de venta de verduras y vestimenta, en fiestas y prácticas deportivas y en el sostenimiento de programas en la radio. Existe otra organización de migrantes, la Asociación de Mujeres Bolivianas de Escobar, entidad mucho más pequeña nucleada en torno a la gestión de recursos, el mantenimiento de prácticas de socialización vinculadas con Bolivia (tejidos, comidas, festividades) y de alfabetización.

En el barrio hay dos escuelas primarias y dos escuelas secundarias públicas y una escuela privada.

En estos diez años recorrí espacios familiares, comunitarios y escolares. En ellos mantuve conversaciones informales, entrevistas, realicé observaciones, participé de diversas situaciones y colaboré con proyectos educativos de las organizaciones y de las escuelas.

En espacios comunitarios inicie los contactos en el año 2011, conversé con referentes de la Colectividad, de la Asociación de mujeres, con madres y padres, niños y jóvenes. Realicé también observaciones de eventos recreativos y asistí regularmente a fiestas cívicas y religiosas. En contextos familiares registré situaciones de interacción cotidiana y conversé con adultos y jóvenes y realicé entrevistas biográficas a mujeres. A partir del año 2013, manteniendo el contacto con espacios familiares y comunitarios comencé a realizar actividades en las escuelas primarias y secundarias. En ellas registre entradas, salidas, clases, actos, converse con docentes directivos y estudiantes, colaboré en la presentación de clases y festividades.

Poniendo en relación esta investigación con otras que previamente había realizando en escuelas de Buenos Aires considero que resulto muy oportuno conectarme primero con las organizaciones y familias y luego con las escuelas. Esto favoreció correrme de la identificación con el rol de maestra o profesora que suelen atribuirnos las familias cuando investigamos temas educativos.

A partir del año 2015 gran parte de este trabajo se desarrolló en colaboración con integrantes del equipo de investigación que coordino en la Universidad de Buenos Aires. Este es un aspecto que también es necesario destacar. Esta colaboración permite abordar la 
problemática desde aspectos diferentes y complementarios: mientras yo me centro en las identificaciones nacionales en contextos festivos comunitarios y en escuelas, otros colegas lo vienen haciendo en espacios laborales y recreativos. El trabajo en equipo fue también fundamental para sostener iniciativas de intervención como un programa de radio en la $\mathrm{CBE}$ (que describo en un punto posterior). Registros conjuntos, materiales audiovisuales compartidos y publicaciones colectivas dan cuenta de las potencialidades del trabajo colaborativo.

Otro aspecto a destacar es el modo en que la metodología debe adecuarse a la intención de reconstruir la riqueza de las situaciones particulares, pero también su atravesamiento por procesos generales. El abordaje local de las problemáticas permite advertir la complejidad de su despliegue, las múltiples dimensiones sociales que las atraviesan, los posicionamientos, las tramas y experiencias de los distintos actores, las disputas de sentido (GUBER, 2001; ROCKWELL, 2009; ACHILLI, 2015); permite también atender a los detalles e interconexiones, el análisis de los hechos en su contexto particular y sin segmentarlos (BALBI, 2020). Pero sabemos que los problemas que abordaremos van mucho más allá de las especificidades de su manifestación territorial. También que el registro de lo local toma sentido en gran parte en vinculación con el contexto social, político y económico mayor.

Desde este presupuesto, el trabajo implicó la complementación de herramientas típicas de la etnografía con otros instrumentos: estudios históricos, relatos biográficos, procesamientos estadísticos (por ejemplo sobre inclusión y exclusión educativa), documentos de distinto alcance (Ley de Educación, Ley de Migración, dispositivos municipales, estatutos de las organizaciones de migrantes, proyectos institucionales de las escuelas, actas de reuniones de las organizaciones, etc.).

El contexto del 2020 impuso nuevos desafíos metodológicos. La búsqueda de contacto y cercanía debieron ser reformuladas por las limitaciones de la movilidad. En esta situación sostener el programa de radio en la Colectividad resulto estratégico para mantener entrevistas telefónicas y virtuales con referentes comunitarios, autoridades educativas, docentes, padres y jóvenes del barrio.

Sintetizando las cuestiones señaladas recupero lo que en mi investigación ha aportado un trabajo de campo intenso y prolongado en el tiempo, la utilización de herramientas tradicionales de la etnografía junto con instrumentos que corresponden a otros enfoques, la labor en equipo y la adecuación a las contingencias y situaciones inesperadas. A continuación propongo reflexionar sobre tres situaciones del trabajo de campo que desde mi punto de vista deben ser consideradas desde un enfoque cualitativo etnográfico: la atención a los sentidos sociales, los alcances de la comprensión, la articulación de actividades típicas de investigación con otras de colaboración e intervención.

\section{Atención a los sentidos sociales y construcción de categorías de análisis}

La antropología tiene una larga tradición en la consideración de los sentidos sociales. Desde los planteos clásicos se miró a las llamadas sociedades etnográficas desde posiciones que permitieron ampliar la forma de entender las instituciones y relaciones familiares, políticas e ideológicas. La marca de la disciplina se asocia a la posibilidad de construir formas de entender las distintas dimensiones sociales corriéndose de lo que las mismas implican en 
occidente. En este punto son indudables los aportes para ampliar la mirada y desnaturalizar el modo en que concebimos la vida social.

Sin embargo, se ha señalado repetidas veces que este ejercicio fue y es relativo, que las posibilidades de descentramiento siempre son limitadas, que no dejamos de ver el mundo desde los parámetros con los que somos formados. También que el trabajo del cientista social no debería acotarse a reconstruir los sentidos que los sujetos dan a su vida y al mundo, sino dirigirse a su interpretación y análisis. En particular que el investigador no debería limitarse a reconstruir lo que los sujetos dicen, sino más bien ponerlo en relación con las practicas y experiencias donde aparecen las categorías en uso y desde allí poner las descripciones en relación con los debates teóricos. Autores como Lahire nos advierten sobre las limitaciones de quedarse con lo que los sujetos expresan sobre si mismos; propone más bien atender a los implícitos, las omisiones de saberes y haceres no legítimos ni objetivados, las inconsistencias entre lo que los sujetos hacen y lo que dicen que hacen (LAHIRE, 2006).

En mi campo de estudio he encontrado particularmente sugerente atender a las categorías locales y procurar tomar distancia de definiciones previas en temas tales como las formas de crianza y familia, la percepción sobre lo que es un niño educado, los saberes válidos en distintos contextos. En torno a estas nociones las expresiones y posicionamientos en el campo invitan a interrogar nuestros posicionamientos previos; ello nos pone ante el desafío de construir categorías más abarcadoras atentas a la diversidad de usos y sentidos.

Entre la población migrante boliviana suele ocurrir que los hijos e hijas sean un tiempo criados entre parientes que viven en Bolivia y en Argentina; también que se afirmen visiones de niñez y juventud distantes de aquellas que se imponen en los medios hegemónicos en Argentina; asimismo, que las organizaciones comunitarias mantengan el protagonismo en la formación de las jóvenes generaciones. De manera complementaria, las expectativas de las familias hacia la escuela se distancian en muchos aspectos de lo que la institución espera y considera legítimo.

En particular me interesa detenerme en algunas categorías centrales para la temática que investigo e imposibles de interpretar sin una mirada atenta a los sentidos que asumen en la localidad: seguir siendo, ser alguien, sangre, bolivianos de segunda generación, descendientes. Desde ya en la población boliviana estos sentidos son coincidentes en algunos aspectos y variables en otros, lo que llama la atención es la recurrencia de su uso.

Un trabajo de campo extendido en el tiempo y que, como dije, combina distintas formas de acceso a la información, permite pensar la centralidad y los sentidos que adquieren estas nociones como parte de las dinámicas sociales de la localidad, más aun, el modo en que las mismas están atravesadas por las tensiones que experimenta la población boliviana en Argentina.

De este modo, numerosas situaciones registradas en contextos familiares y comunitarios dan cuenta del proyecto colectivo por sostener la continuidad como bolivianos; también de como lo mismo se transforma en un mandato hacia las jóvenes generaciones. Que sus hijos sigan siendo bolivianos, que la sangre opere como marcador de pertenencia, que participen en prácticas (laborales, festivas, recreativas) sostenidas por las organizaciones de migrantes son componentes fundamentales de un proyecto de reproducción colectiva en un contexto de movilidad y subordinación (NOVARO, 2015). 
Desde la recuperación de estos sentidos me parece necesario discutir con algunos investigadores que ven en estas nociones (segunda generación, hijos de, descendientes) como un mecanismo de la nueva sociedad para acentuar situaciones de marcación y discriminación. Esta discusión por supuesto no implica negar estos aspectos, pero si advertir la necesidad de que estas tendencias dialoguen con los sentidos que los propios colectivos dan a estas adscripciones. Desde la consideración de los mismos nos enfrentamos al desafío de un ejercicio espiralado de recolección de información, registro de interconexiones, reflexiones teóricas situadas y afinamiento de las categorías.

\section{Comprensión y empatía}

Las relaciones en el campo nos ponen ante innumerables situaciones, en muchas de ellas sentimos una gran cercanía con los sujetos, en otras no tanto. Cuando las posiciones de los sujetos con las que empatizamos y con las que no refieren a los temas que trabajamos, resulta necesario atender a nuestras miradas y el modo en que manejamos la proximidad y la distancia.

Me he encontrado muchas veces ante circunstancias con las que sentí incomodidad e incluso que ponían en tensión mis presupuestos éticos. Voy a mencionar dos de estas situaciones vinculadas al tema que investigo: las expectativas de las familias sobre sus hijos en clave de disciplinamiento y las representaciones de las escuelas sobre las familias bolivianas.

En espacios familiares y comunitarios he registrado la preocupación de los padres bolivianos porque sus hijos mantengan la imagen de niños educados asociada a Bolivia. En estas proyecciones se refirma la asociación de lo boliviano con atributos como respetuoso, trabajador, obediente. En coincidencia muchas familias bolivianas dicen esperar que en las escuelas argentinas haya más disciplina y orden, se respete la autoridad adulta; resulta también recurrente la referencia añorada a las formas escolares más disciplinadas en Bolivia ${ }^{2}$. Tanto las imágenes de niñez como la demanda de disciplina y orden a la escuela aparecen asociados a la búsqueda de que sus hijos reproduzcan modos de ser y comportarse como niños bolivianos.

Me resulto muy complejo distanciarme de mis propios presupuestos sobre la niñez, crianza, los derechos y los estilos supuestamente democráticos de interacción para analizar estas expectativas. Fue necesario todo el tiempo tener presente la diferencia entre el intento de comprenderlas y la adhesión a ellas.

Distintos elementos fueron permitiendo verlas desde múltiples aristas: la responsabilidad de los niños en los rituales y las actividades productivas en las comunidades andinas que distintos autores han señalado (ARNOLD y YAPITA de DIOS, 2005; AMES, 2013), el formato religioso y militar que a pesar de las reformas educativas de los últimos años sigue atraveando a las escuelas en Bolivia, sobre todo en la zona rural. Sumado a esto, la relevancia de los niños y jóvenes en el proyecto migratorio, la apuesta porque sus hijos sean alguien en la vida asociada a una escolaridad larga y la tendencia a la idealización del territorio dejado entre los adultos migrantes.

\footnotetext{
${ }^{2} \mathrm{Si}$ bien estas posiciones son recurrentes, también es importante destacar que no son únicas ni uniformes. He registrado también padres y madres que critican el carácter autoritario, la rigidez y la imposición de la sumisión en las escuelas de Bolivia y valoran el trato afectivo y personalizado de las escuelas argentinas.
} 
Debí también atender a la tensión explicita con el modelo de niño y educación de las instituciones argentinas en tanto las mismas, al menos discursivamente, se sostienen en un paradigma de respeto a los derechos del niño. Entiendo por todo esto que la demanda de disciplinamiento de las familias bolivianas debe ser interpretada como parte de la apuesta porque las jóvenes generaciones se socialicen en pautas similares a las que vivieron sus antecesores. En definitiva, para comprender los sentidos de esta demanda es necesario considerar las tensiones identitárias en la localidad, concibiendo a la escuela como un espacio más donde se manifiesta la apuesta de familias y organizaciones por la continuidad y la experiencia de la discontinuidad en las relaciones intergeneracionales (NOVARO, 2015).

La otra situación con la que quiero ejemplificar la necesidad de distinguir la comprensión de la adhesión se refiere a las representaciones docentes sobre las familias bolivianas.

En las escuelas advierto la voluntad de muchos docentes por tender puentes y la presencia de un discurso de inclusión e interculturalidad, pero también limitaciones de las instituciones para dejar de asociar la diversidad con la carencia, para poner en duda los propios presupuestos y desde allí dialogar con colectivos que se alejan de los parámetros con los que se estructuró el sistema educativo. En cuestiones como las identificaciones nacionales, las formas de participación de las familias, los conocimientos considerados válidos, los cambios han sido relativos. En particular persisten concepciones sobre las familias bolivianas que las responsabilizan por los problemas de la escolaridad, enfatizan y generalizan su carácter "cerrado": "solo se juntan entre ellos", "no participan ni responden" "la escuela no es una prioridad", suelen ser comentarios reiterados por los docentes. También registro el desconocimiento de los actores escolares de las experiencias formativas que los niños y jóvenes realizan en espacios familiares y comunitarios. Revisar estas miradas y omisiones parece una condición imprescindible para trabajar con la condición transnacional de vida de los niños y jóvenes que habitan las escuelas.

Comprender las razones de la persistencia de concepciones tradicionales implica verlas en su dimensión histórica, atender al carácter gradual y contradictorio de las transformaciones, ubicar a las escuelas en relación a las tensiones del contexto social y político. Todo ello no implica justificar la persistencia de preconceptos discriminatorios, en tanto continúan operando como mecanismos de exclusión educativa.

Estas situaciones (sumadas a otras similares) y muchas dudas desde las que sigo interrogando el campo nos interpelan sobre la posibilidad de comprender miradas y practicas a las que no adherimos, pero que creo necesario no juzgar precipitadamente desde nuestros presupuestos morales, sobre todo cuando esos juicios obturan la posibilidad de entenderlas en relacion a su contexto y en toda su complejidad.

Resultan en este punto necesarias algunas aclaraciones en torno al término comprensión. Comprender es aquí entendido como tornar inteligible, como entender las razones de las palabras, acciones y marcos conceptuales que no son los propios, la lógica de situaciones que contrarían nuestros presupuestos. Resulta útil aquí reponer los términos de Geertz acerca de la importancia de no olvidar la diferencia entre la comprensión y la empatía; en este sentido afirma que comprender no es alcanzar la comunión con los nativos y que hacer etnografía es una empresa que suele presentar "perplejidades morales" (GEERTZ, 1990, p. 372). Asocia la comprensión a una traducción de sistemas simbólicos y se pregunta que sucede cuando "ya no 
podemos pretender una identificación transcultural con nuestro objeto" (GEERTZ, 1994, p.74). Este autor en definitiva aboga por producir una interpretación que no sea ni prisionera de los horizontes mentales del contexto donde se expresan los hechos, pero tampoco ajena a sus existencias (GARCIA AMIBURU, 2000)

Estas precisiones resultan sugerentes para poder pensar en las situaciones que analizo (las demandas de disciplinamiento, las visiones escolares de las familias bolivianas), distantes en muchos sentidos de los marcos teóricos, ideales políticos y valoraciones que solemos explicitar.

Siempre parece necesaria cierta toma distancia (sabiendo los alcances parciales de ello y sin ninguna pretensión de neutralidad), como paso necesario para la reflexividad critica, pero también una cuota importante de flexibilidad para advertir "lo que para uno no tiene sentido" (GUBER, 2001, p. 17) y, sobre todo, lo que contraria nuestros sentidos y valores.

En los temas vinculados a la educación y formación de las jóvenes generaciones, considerando nuestras referencias nacionales, de clase y formación, cabe la advertencia sobre la necesidad de entender pero también tomar distancia de las visiones de los docentes sobre las familias migrantes pobres. Muchas investigaciones toman como dato de campo lo que en verdad son apreciaciones de las instituciones escolares sobre realidades y sujetos que se alejan de los parámetros esperados por el sistema.

En definitiva, explicitar y reflexionar sobre los presupuestos en los que hemos sido formados, parece una condición para no profundizar distancias, habilitar la escucha de lo que "el otro" expresa y representa, y dejar, de alguna manera, que la mirada del otro "nos alcance" (SEGATO, 2013, p. 12). En este proceso sabemos que la mirada del otro no necesariamente coincidirá con la nuestra, pero también sabemos que es posible que sus miradas y sus voces sacudan nuestros presupuestos y los enriquezcan.

\section{Etnografía, intervención y reflexividad}

Desde hace años en las ciencias sociales se plantea el debate sobre la forma en que damos respuesta a las demandas que los actores nos plantean en el curso de nuestros trabajos. También se señala la relevancia de compartir y divulgar los resultados de las investigaciones fuera de la academia. Esto implica un replanteo de paradigmas que presuponen la objetividad, la neutralidad y el no involucramiento como condición de la producción de conocimiento. Lo mismo se enmarca en los debates sobre la investigación acción (FALS BORDA, 1990) y la etnografía colaborativa (RAPAPPORT, 2007). Estas propuestas han sido debatidas en distintos aspectos: el presupuesto de simetría en las relaciones de investigadores y colectivos que suelen ser inherentemente asimétricas, las formas de validación de un saber focalizado en los efectos de la intervención, las dificultades para mantener distanciamientos necesarios de las tensiones y conflictos en el campo, etc. No obstante acordar con la necesidad de considerar estos aspectos, desde mi punto de vista las propuestas de investigación acción e investigación colaborativa, continúan interpelando las formas tradicionales de producción y legitimación del conocimiento científico.

Desde este presupuesto y considerando los reparos señalados, relato tres situaciones de intervención desarrolladas en la localidad de Escobar y destaco el modo en que las mismas 
aportaron a las formas de relación y a pensar los temas de investigación. La primera y la última fueron actividades colectivas.

Las actividades de colaboración se realizaron en principio con las escuelas, en el caso de las escuelas secundarias se hicieron a partir del 2015 en colaboración con María Laura Diez. Desde el inicio de la investigación sostuvimos el apoyo en el dictado de talleres con docentes y estudiantes, colaboramos en las secuencias de las clases que observábamos compartimos material con los docentes e incluso nos hicimos cargo de alguna parte de las secuencias. Estas situaciones fueron generando mucha empatía en los espacios escolares, permitieron más proximidad, nos corrieron del lugar de observadores y sobre todo de la asociación que tiende a hacerse de nuestro rol con la supervisión de la tarea docente. También hubo situaciones complejas sobre las que es necesario seguir profundizando. En algunos momentos nuestro lugar estuvo atravesado por conflictos internos de la escuela, tensiones personales y manejos profesionales que hicieron necesaria la búsqueda de un lugar preservado de ciertos conflictos pero comprometido con las iniciativas que creímos direccionadas por voluntades de inclusión Siempre compartimos con las escuelas informes escritos de las actividades realizadas. Resulta todo un desafío encontrar el tono y el estilo de estos textos, comentar reflexiones críticas sin que las mismas se asocien a la descalificación. También debemos reflexionar sobre situaciones de este tipo que nos generan conflictos en el campo y sincerar que en muchas ocasiones no podemos manejar estos conflictos; solo a modo de ejemplo, a principios del año 2015 mis informes de devolución del trabajo realizado en las escuelas primarias tuvieron efectos inesperados al llegar a la inspección educativa: daba en ellos cuenta de las condiciones deficitarias en infraestructura y equipamiento de una de las escuelas del barrio (la llamada por muchos "la escuela de los bolivianos") y de que la misma era evitada por gran parte de la población de la localidad. Asimismo, mi cercanía con una asistente social de origen boliviano que trabajaba en esta institución (con la que realizamos actividades y escribimos textos conjuntos) no fue bien vista por la directora que tenía una relación conflictiva con ella. Luego de algunos intentos infructuosos de reconstruir la confianza seguí trabajando en otras instituciones escolares del barrio.

Otra experiencia de intervención que enriqueció la investigación fue la colaboración con un proyecto educativo de la Asociación de Mujeres Bolivianas de Escobar. Cuando en el 2012 conocí a la presidenta de la entidad me solicito apoyo para organizar una escuela en su domicilio. Fundamentó la importancia de este proyecto con argumentos significativos considerando las temáticas de mi interés. Además de aludir a su "vergüenza" porque sus paisanas no sabían firmar, comentó que saber escribir facilitaría que las mujeres ayuden a sus hijos en la escuela. El funcionamiento de un centro educativo en la asociación se institucionalizo en el 2014 y continúo hasta la pandemia. Mi colaboración en el armado del proyecto y la concreciona de contactos con referentes del sistema educativo fue muy valorada. En este periodo pude realizar reconstrucciones de trayectorias de varias mujeres de la asociación, fui también objeto de demandas más personales por cuestiones como la crianza de los niños, temas legales, etc.

Posteriores situaciones en el barrio y reformulaciones en la Asociación también me hicieron reflexionar sobre las implicancias de este tipo de proyectos que ayudamos a sostener, tal vez desde presupuestos simples que asocian más educación con más inclusión. 
El funcionamiento de la asociación de mujeres como un centro de alfabetización y su reconocimiento como centro educativo coincidió con reformulaciones en su composición y funcionalidad. Se incluyó en las actividades de la escuela a población sin adscripción al colectivo boliviano; al mismo tiempo la presidenta profundizó el contacto con distintos referentes políticos locales. Distintas situaciones colaboraron a que se diluyera su asociación con un espacio de sociabilidad comunitaria de las mujeres bolivianas y descendientes.

Por último y siempre buscando ejemplificar la potencialidad y también la complejidad de incluir actividades de intervención en la investigación daré cuenta de lo que implicó para el compromiso de sostener un programa de radio en la emisora de la Colectividad ${ }^{3}$.

El programa se originó en una demanda del secretario de comunicación de la CBE luego de que visitáramos la radio repetidas veces. En una ocasión asistí con la presidenta de la Asociación de Mujeres a la radio y al finalizar el encuentro, el operador y el subsecretario de comunicación me dijeron: "Ustedes estaban por venir a la radio con la escuela [...] porque nosotros queremos dar un lugar para lo educativo, estaría bueno que vinieran... Podrían tener un programa todas las semanas", (Registro 15 de abril de 2019). El programa comenzó a emitirse en el 2019 y continúa hasta la actualidad. Es como antes decía una experiencia sostenida colaborativamente por el equipo de investigación que coordino en la localidad.

El espacio se transformó en una instancia de diálogo sumamente enriquecedora constituyéndose en uno de los puentes que desde hacía años procurábamos construir entre las organizaciones y las escuelas. Nos propusimos visibilizar las experiencias educativas que se desarrollan en la $\mathrm{CBE}$, recuperar recuerdos escolares de Bolivia y Argentina y pensar la escolaridad en un contexto de movilidad. En paralelo, permitió que en el medio de difusión fundamental de la Colectividad se escuchara el testimonio de directivos y docentes del barrio. Los testimonios recordaron con nostalgia experiencias en las comunidades rurales, también el sufrimiento de los primeros años de arribo a Argentina, escenas escolares de discriminación, otras de inclusión y de docentes que tendieron puentes. En el 2020, múltiples voces relataron las complejidades de continuar la escolaridad para la población migrante en condiciones de pobreza.

Para muchos docentes que invitamos a la radio esta era la primera vez que visitaban los edificios de la Colectividad. Considerando su antigüedad en el barrio y las escuelas esto es a la vez dato para la investigación (en tanto indicio de la distancia entre las escuelas y las organizaciones) y objeto de la intervención.

La participación en el programa reforzó los lazos del equipo con la Colectividad y con las escuelas, permitió el tránsito por situaciones antes poco imaginables, naturalizó nuestra presencia en múltiples eventos.

Como en las experiencias anteriores, sin duda en esta también se presentarán dilemas y efectos contradictorios. La distancia impuesta por el aislamiento social tal vez no nos este permitiendo registrar situaciones complejas que será necesario pensar desde nuestras investigaciones y rectificar desde los dispositivos de intervención. Al día de hoy, con el programa en curso, parece necesario reflexionar sobre lo que implica que los temas educativos y escolares sean tratados en la radio de la Colectividad por sujetos ajenos a la misma y que

\footnotetext{
3 En un texto reciente desarrollamos con detalle esta experiencia (Diez, Novaro, Fariña, Ferreiro y Varela, en evaluación)
} 
efecto tiene esto en una organización atravesada por una lógica muy compleja de afirmación de pertenencias propias y lucha por la inclusión en Argentina.

\section{Palabras finales}

A partir de las iniciativas de colaboración-intervención recién comentadas profundicé la reflexión sobre temas como la situación de las escuelas en contextos transnacionales, las formas de relación de las organizaciones de migrantes con instituciones del estado argentino, las trayectorias escolares en Bolivia y en Argentina, las experiencias formativas de los jóvenes en la Colectividad. Estas actividades también me hicieron pensar en el compromiso con cierta expectativa de reciprocidad en la vinculación de los colectivos con los que trabajamos. Por ello considero que las actividades de colaboración-intervención, lejos de entenderse como distintas a la investigación, son parte de ella en tanto suponen formas de acceso a la información, profundización de relaciones y ocasiones para la reflexión que aportan a ajustar los proyectos y el análisis.

De esta forma, los tres temas abordados en este texto (la atención a las categorías sociales, la comprensión y la inclusión de actividades colaborativas) se unen en la intención de pensar formas de producción de conocimiento atentas a los sentidos locales, a la necesidad de entender situaciones aun cuando no adherimos a ellas (tal vez, sobre todo porque no adherimos a ellas), a involucrarnos en iniciativas importantes para los sujetos con los que trabajamos aun cuando nos corran de las actividades que inicialmente planeamos en nuestros proyectos.

Todo esto de ninguna manera implica que sea posible y deseable trabajar sin conceptos previos, principios morales o esquemas planificados. Significa en cambio aceptar que nuestras categorías, principios y planes al tiempo que nos orientan, deben tener la suficiente flexibilidad como para ser interpelados por las realidades que estudiamos.

\section{Bibliografía}

ACHILI. Elena. Investigar en antropología social. Los desafíos de transmitir un oficio, Rosario, Argentina: Laborde Editor, 2015

ARNOLD, Denise, y YAPITA DE DIOS, Juan. El rincón de las cabezas. Luchas textuales, educación y tierras en los Andes. La Paz, Bolivia: Universidad Mayor de San Andrés, ILCA, 2005.

AMES, Patricia. Niños y niñas andinos en el Perú: crecer en un mundo de relaciones y responsabilidades. Bulletin de l'Institut français d'études andines. Francia, v. 42, n. 3, 389-409, 2013.

BALBI, Fernando. La inversión de la teoría en la etnografía en antropología social. Revista del Museo de Antropología, Córdoba, Argentina, v. 13, n 2, p. 203-214, 2020.

BARTOLOMÉ, Miguel. En defensa de la etnografía: aspectos contemporáneos de la investigación intercultural. Anuário Antropológico, V. 28, $N^{\circ}$ 1, p. 29-60, 2003.

FALS BORDA, O. [et al.] Acción y conocimiento, cómo romper el monopolio con investigación-acción participativa; traducción Raquel González. Bogotá: Cinep, 1991.

GARCIA AMILBURO, Maria. La comprensión del otro. ¿Empatía o traducción? Emociones Themata, $\mathrm{N}^{\circ} 25$, p. 209-215, 2000. 
GEERTZ, Clifford, La interpretación de las culturas. Barcelona: Gedisa, 1990.

GEERTZ, Clifflord. Conocimiento local. Ensayos sobre la interpretación de las culturas. Barcelona: Paidos, 1994.

GUBER, R. La etnografía, Método, campo y reflexividad. Buenos Aires: Ed. Norma, 2001.

LAHIRE, Bernard. El espíritu sociológico. Buenos Aires: Manantial, 2006.

NOVARO, Gabriela. "Ellos llevan a Bolivia en la sangre". Transmisión intergeneracional en contextos de migración y pobreza. Horizontes Sociológicos. Buenos Aires, $\mathrm{n}^{\circ}$ 6, p. 37-53, 2015.

RAPPAPORT, J. Más allá de la escritura: La epistemología de la etnografía en colaboración. Revista colombiana de antropología, v 43, p. 197-229, 2007.

ROCKWELL, Elsie. La escuela cotidiana. Fondo de Cultura Económica: México, 1995.

SEGATO, R. (2013). La crítica de la colonialidad en ocho ensayos y una antropología por demanda. Ciudad Autónoma de Buenos Aires: Prometeo Libros, 2013. 